Polymer Journal, Vol. 38, No. 9, pp. 905-911 (2006)

(C) 2006 The Society of Polymer Science, Japan

\title{
Long Term Water Adsorption Ratio Improvement of Polypropylene Fabric by Plasma Pre-treatment and Graft Polymerization
}

\author{
Ko-Shao Chen, ${ }^{1, \dagger}$ Hong-Ru Lin, ${ }^{2}$ Su-Chen ChEn, ${ }^{1}$ Jui-Che TsAi, ${ }^{1}$ and Yuan-An Ku ${ }^{3}$ \\ ${ }^{1}$ Department of Material Engineering, Tatung University, TAIWAN, R. O. C. \\ ${ }^{2}$ Department of Chemical Engineering, Southern Taiwan University of Technology, TAIWAN, R. O. C. \\ ${ }^{3}$ Functional Treatments Section, Department of Product Development, \\ Taiwan Textile Research Institute (TTRI), TAIWAN, R. O. C.
}

(Received December 5, 2005; Accepted May 23, 2006; Published July 28, 2006)

\begin{abstract}
Although the hydrophilicity of polypropylene (PP) fabric surface can be improved by plasma treatment, it would obviously decay with the storage time. In this study, the graft polymerization of acrylamide (AAm) onto the surface of PP fabric was carried out by plasma pre-treatment and subsequently induced by UV-induce surface graft polymerization to improve the long term water adsorption ratio of the substrate. During the process, peroxides formed by the plasma treatment are the major factor responsible for initiating the graft polymerization. The amount of peroxide can be determined with good accuracy by the 1,1-diphenyl-2-picrylhydrazyl (DPPH) method. Results indicated that the amounts of peroxides obviously increased with the plasma treated time and treat power, and the maximum concentration of peroxide can reach about $6.0 \times 10^{-7} \mathrm{~mol} / \mathrm{cm}^{2}$. However, the degree of grafting was not monotonous with increasing plasma treated power. The optimum processing condition was to apply $30 \mathrm{~W}$ power with $10 \mathrm{~min}$ of treatment to achieve the maximum surface grafting efficiency. Although the surface of PP fabric can be changed from hydrophobic to hydrophilic after plasma treatment, it would obviously age after $4 \mathrm{~d}$ of storage. By contrast, the UV-induce surface graft polymerization of PP can keep their hydrophilic property during the storage time after $7 \mathrm{~d}$, due to the covalently grafted with hydrophilic monomer. [doi:10.1295/polymj.PJ2005183]

KEY WORDS Plasma Treatment / Graft Polymerization / Water Adsorption Ratio Improvement / Peroxide Determined /
\end{abstract}

Polypropylene (PP) fabric was widely used due to its cheapness, low density, excellent mechanical properties, and high porosity. However, the inert surface and lack of chemical polar groups of hydrophobic polymers, restricts their applications in some fields such as sanitary products, dressing, and biomaterials. ${ }^{1-5}$ Thus, several methods have been developed to modify surfaces of these polymers to improve their water adsorption ratio. ${ }^{6-9}$

In which, the use of inner gas plasma treatment has been extensively studied to modify surface properties of polymer substrates. Free radicals are formed on the surface of substrate after plasma treatment. Peroxides are also generated at the same time when the free radicals are exposed to air. ${ }^{10}$ Thus, the water adsorption ratio of the surface exhibits the obviously decay effect during the storage time due to the reorientation of the hydrophilic group toward the bulk of the polymer. ${ }^{11}$ To achieve a stable modification surface, subsequently graft polymerization utilizing free radicals or peroxides generated by the plasma treatment was extensively adopted by many studies. ${ }^{12-14}$

The plasma composed of inorganic gas argon leads to implantation of atoms, radical generation, and etch- ing reaction. There have been published quite a large number of works on the polymer surface modification by graft copolymerization utilizing free radicals or peroxide generated by the plasma treatment. So far, very few works have been reported on the relationship between the concentration of peroxides generated and the degree of graft co-polymerization. In this study, argon plasma activation and photo-induced graft polymerization of acrylamide (AAm) monomer onto PP fabric was conducted to improve the long term water adsorption ratio of the substrate. The amounts of the surface peroxides after plasma treatment were determined and quantified by 1,1-diphenyl-2-picrylhydrazyl (DPPH) method. ${ }^{15-17}$ The present work was concentrated on the relationship between the concentration of peroxides generated and the degree of graft copolymerization. In addition, the optimum conduction on water adsorption ratio of PP fabric was also discussed. The reaction conditions such as plasma treated time and plasma supplied power were evaluated. The water adsorption ratio of PP fabric after modification was assayed by the water absorption ratio and the chemical composition after surface grafted with AAm was also analyzed and confirmed by electron spectroscopy.

${ }^{\dagger}$ To whom correspondence should be addressed (Tel: 886-2-25867150, Fax: 886-2-25936897, E-mail: kschen@ttu.edu.tw). 


\section{EXPERIMENTAL}

\section{Materials}

PP fabric (cut into the size: $2 \times 3 \mathrm{~cm}^{2}$ ) was chosen as the substrate. Before treatment, the substrate was washed by alcohol overnight to remove the residual substance. Acrylamide (AAm) $\left(\mathrm{C}_{2} \mathrm{H}_{5} \mathrm{CONH}_{2}\right)$ monomer was purchased from Wako chemical company. Riboflavin $\left(\mathrm{B}_{2}, \mathrm{C}_{17} \mathrm{H}_{20} \mathrm{~N}_{4} \mathrm{O}_{6}\right)$ adopted to reduce the oxygen concentration during the polymerization process was purchased from Sigma Chem. Company. In addition, 1,1-diphenyl-2-picrylhydrazyl (DPPH) purchased from Wako Pure Chemical Industry was used to determine the amount of peroxide after plasma treatment.

\section{Plasma Activation}

The equipment of plasma treatment is shown in Figure 1. PP fabric was individually placed on the surface of lower electrode. The plasma system (Model PD-2S) manufactured by SAMCO, Japan, was first pumped to a base pressure of $5.33 \mathrm{~Pa}$ before plasma treatment. When the vacuum index was stable at $5.33 \mathrm{~Pa}$, the argon gas was continuously introduced into the reactor and adjusted to working pressure $(26.66 \mathrm{~Pa})$ for a stable period of time. Then the plasma was activated by RF generator $(13.56 \mathrm{~Hz})$, with a treating time of $0.5-10 \mathrm{~min}$ and a plasma power of 5-70 W.

\section{Concentration of Free Radicals on PP Surface}

After the plasma treatment, free radicals were deposited onto the surface of the PP fabric. Once the substrate was taken out and exposed to air, the activated radicals immediately reacted with oxygen to form peroxides on the surface. In order to quantify these peroxides, DPPH assay was found to be an effective

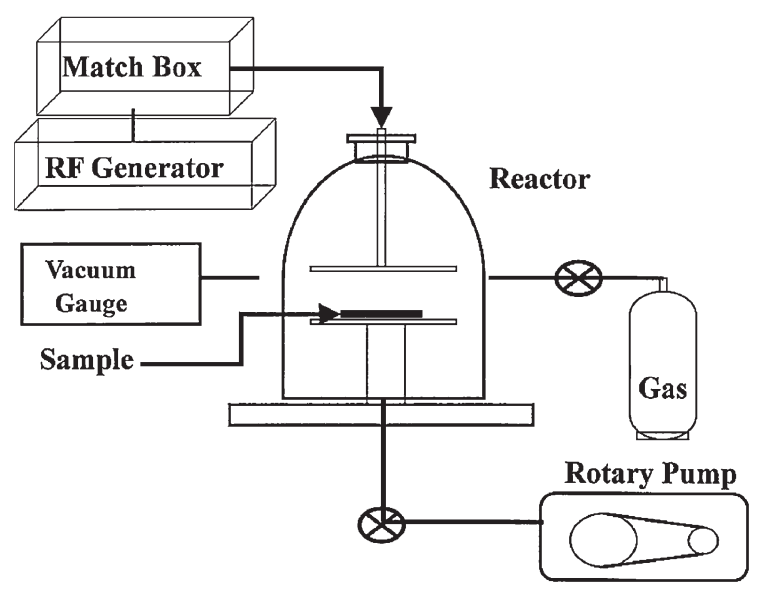

Figure 1. The schematic diagram of argon plasma treatment apparatus. method. Substrate was set in atmosphere for $24 \mathrm{~h}$ before being subjected to DPPH solution. It is believed that all the free radicals were turned to peroxides during this period of time. Substrate was put into the DPPH $\left(1.0 \times 10^{-4} \mathrm{~mol} \mathrm{~L}^{-1}\right)$ solution in a glass ampoule and kept at $70^{\circ} \mathrm{C}$ overnight to throughout decompose the peroxides. The DPPH molecules were consumed by the decomposition of peroxides and can be measured by UV spectrophotometer at $520 \mathrm{~nm}$. The absorption coefficient of pure DPPH at $520 \mathrm{~nm}$ is $1.18 \times 10^{4} \mathrm{~L} / \mathrm{mol}-\mathrm{cm}$. In this method, the calibration curve was created and used to determine the unknown peroxides concentration of each sample.

\section{$U$-induced Graft Polymerization}

After $10 \mathrm{wt} \%$ AAm aqueous solution was prepared, $0.01 \mathrm{~g} / \mathrm{L}$ riboflavin $\left(B_{2}\right)$ was subsequently mixed with the AAm solution in the ratio of AAm:riboflavin = 4:1. The PP fabric was immersed into the AAm solution in the Pyrex glass container. This container was sealed and then irradiated with $1000 \mathrm{~W}$ UV light (wavelength: $365 \mathrm{~nm}$, Henchman Co. Inc., Taiwan) for $30 \mathrm{~min}$. After the graft polymerization reaction, the homopolymer of PAAm was removed by washing in distilled water at $70^{\circ} \mathrm{C}$ for $24 \mathrm{~h}$ under continuous stirring.

\section{Characterization}

Graft Yield. The graft yield was calculated from the increased in weight of the substrate after graft copolymerization as follows:

$$
\text { Graft yield }(\%)=\left[\left(\mathrm{W}_{2}-\mathrm{W}_{1}\right) / \mathrm{W}_{1}\right] \times 100 \%
$$

Where $\mathrm{W}_{1}$ and $\mathrm{W}_{2}$ denote the weights of substrate and the grafted substrate, respectively.

Scanning Electron Microscopy (SEM). The surface morphology of modified fabric was observed from SEM (Hitachi S-800 and JSM-6300). The surface of fabric was first coated with gold films by vacuum evaporated of gold, which made the specimens surface as better conductors and decreases beam damage.

Fourier Transform Infrared (FT-IR) Spectroscopy. Attenuated Total Reflection (ATR, 300E, JASCO) spectroscopy was used to confirm chemical characterization of surface modified membranes. The incident angle of infrared light against the prism was $45^{\circ}$ and the scanning range was 4000 to $400 \mathrm{~cm}^{-1}$.

Electron Spectroscopy for Chemical Analysis (ESCA) Spectroscopy. The elemental composition at the polymer surface was determined by ESCA spectra (PHI 590AM, Perkin-Elmer) using $\mathrm{MgK} \alpha$ exciting radiation. Typical operating conditions were X-ray gun, $15 \mathrm{kV}, 250 \mathrm{~W}$, the pressure of the sample chamber was $1.3 \times 10^{-8} \mathrm{~Pa}$ and the take-off angle was $54.7^{\circ}$. 
Water Adsorption Ratio Test. The water adsorption ratio for surface modified fabric in water was calculated from the increased in weight of the fabric after immersing in water as follows:

$$
\begin{array}{r}
\text { The water adsorption ratio }(\%) \\
=\left[\left(\mathrm{W}_{2}-\mathrm{W}_{1}\right) / \mathrm{W}_{1}\right] \times 100 \%
\end{array}
$$

Where $\mathrm{W}_{1}$ and $\mathrm{W}_{2}$ denote the weight of the fabric and fabric immersed in water, respectively.

\section{RESULTS AND DISCUSSION}

\section{Effect of Plasma Treatment Condition on the Genera-} tion of Peroxides and Degree of Grafting

Y. Ikada ${ }^{10}$ indicated that at least two contradictory processes, radical formation and recombination, occur during the plasma irradiation. When polymer exposed to plasma, hydrogen abstraction will occur from the surface of polymer to produce radicals $(\mathrm{P} \cdot$ and $\mathrm{H} \cdot)$ (eq 1). The resulting polymer radical will react with another polymer radical to from cross-linking (eq 2) or will react with oxygen in air (eq 3) to produce peroxide when the polymer is taken out to air.

$$
\begin{aligned}
& \mathrm{PH} \rightarrow \mathrm{P} \cdot+\mathrm{H} \cdot \\
& \mathrm{P} \cdot+\mathrm{P} \cdot \rightarrow \mathrm{P}-\mathrm{P} \\
& \mathrm{P} \cdot+\mathrm{O}_{2} \rightarrow \text { Peroxide }
\end{aligned}
$$

When polymer is surface treated by plasma and is stored in air, most of the free radicals of the polymer eventually must be converted to peroxide. The DPPH technique was utilized to evaluate the concentration of peroxide formed in the irradiated PP fabric by measuring the quantity of DPPH consumed from the reaction of peroxide radicals with DPPH. The principle of this method is to measure the consumption of DPPH when the plasma treated fabric is put in a benzene solution containing a given amount of DPPH to decompose the peroxides. It is believed that more consumption of DPPH, more decomposition of peroxides, and more free radicals were originally formed on the surface of substrate after plasma treatment. Figure 2 shows the decomposed peroxide concentration calculated from the DPPH consumption as a function of the reaction time at $70^{\circ} \mathrm{C}$ for the PP fabric exposed to Ar plasma $(50 \mathrm{~W})$ for $10 \mathrm{~min}$. The decomposition of peroxide continued for up to $20 \mathrm{~h}$ and then leveled off. Therefore, the concentration of peroxide formed on the irradiated PP fabric was determined after decomposing the peroxides in the DPPH solution at $70{ }^{\circ} \mathrm{C}$ for $24 \mathrm{~h}$.

Figure 3 illustrated the effect of Ar plasma treated time on radical concentration and degree of PAAm grafted onto PP fabric. The concentration of free radicals as a function of plasma treated time was de-

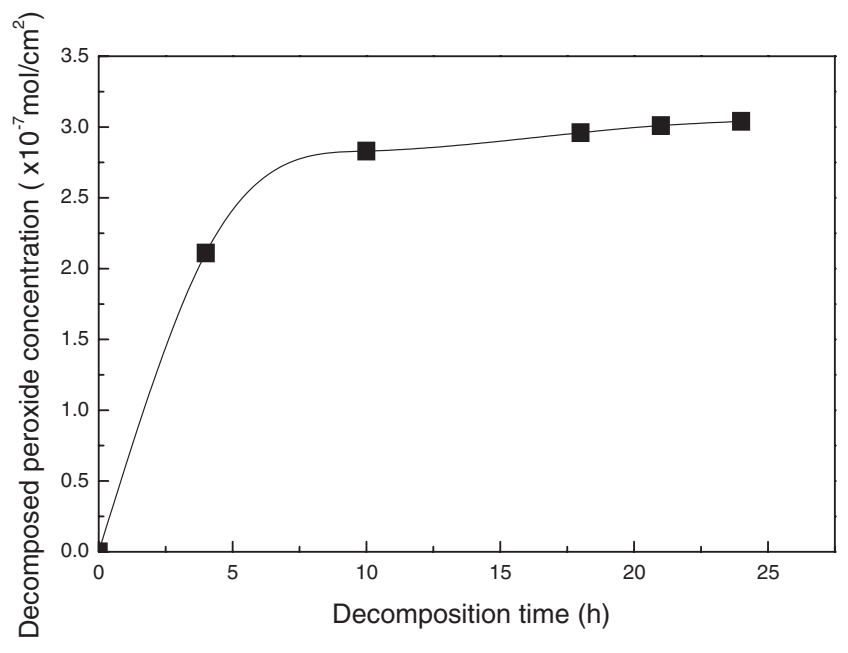

Figure 2. The decomposed peroxide concentration as a function of reaction time in DPPH solution at $70^{\circ} \mathrm{C}$ (Plasma treatment: $50 \mathrm{~W}, 10 \mathrm{~min}$ ).

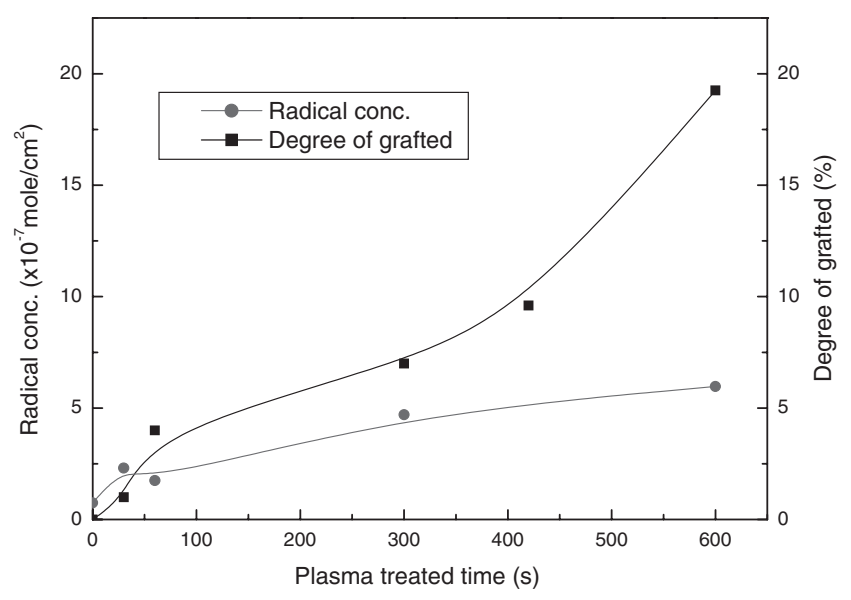

Figure 3. The effect of Ar plasma treated time on radical concentration and degree of PAAm grafted onto PP fabric (Plasma treatment: $50 \mathrm{~W}$ ).

termined indirectly from the consumption of DPPH. Apparently, in these experiment conditions, the concentration of surface free radicals not yet reached the maximum amount, so the transition point was not seen in the figure. The result was different from those reported by Y. Ikada et al. ${ }^{10}$ they indicated that the peroxide concentration at the surface of the plasma-irradiated polyethylene film increased with an increase in the plasma irradiation time, went through the maximum, and then decreased. The free radicals eventually remaining in the surface region of the fabric were converted to peroxides during the storage; these peroxides can subsequently initiate the graft polymerization reaction at high-temperature circumstance. The trend of the relationship between plasma treated time and grafting degree of PAAm on PP fabric was in good agreement with the results shown in concentration of free radicals. The degree of PAAm 


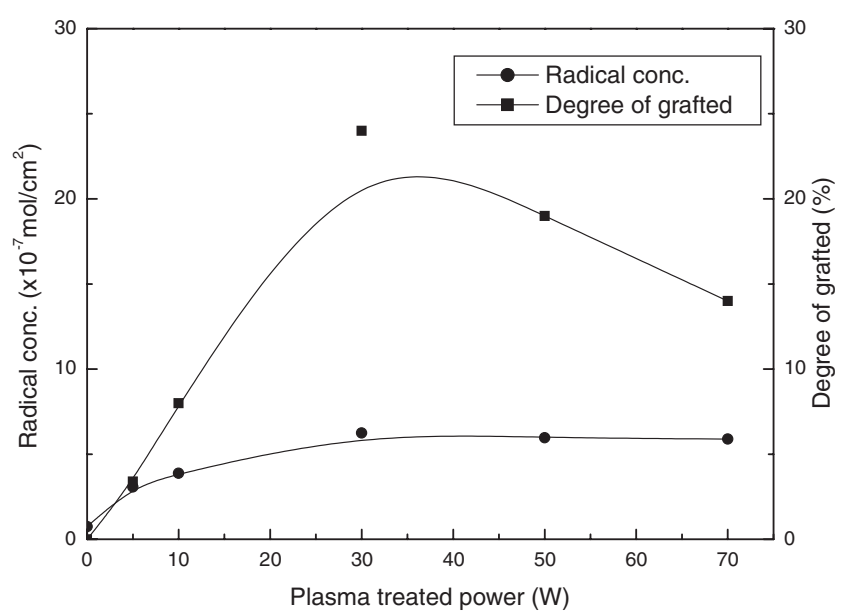

Figure 4. The effect of Ar plasma treated power on radical concentration and degree of PAAm grafted onto PP fabric (Plasma treatment: $10 \mathrm{~min}$ ).

grafted increased with treated time due to higher concentration of peroxides were formed and more of grafting sites were created. The maximum value of degree of PAAm grafted was about 20\% at Ar plasma treatment $10 \mathrm{~min}$. Figure 4 illustrated the effect of $\mathrm{Ar}$ plasma treated power on radical concentration and degree of PAAm grafted onto PP fabric. The concentration of free radicals as a function of plasma treated power was determined indirectly from the consumption of DPPH. The result is similar to those obtained from changing of plasma treated time. The free radicals concentration was obviously increased with increasing the treated power at $30 \mathrm{~W}$ then leveled off, and the maximum value was about $6 \times 10^{-7} \mathrm{~mol} /$ $\mathrm{cm}^{2}$. However, the degree of PAAm grafted increased before $30 \mathrm{~W}$ and then dropped beyond $30 \mathrm{~W}$, this may be due to the slightly decrease in concentration of free radicals after $30 \mathrm{~W}$. The maximum value of degree of PAAm grafted was $24 \%$.

Therefore, a better choice on experimental condition was determined as $30 \mathrm{~W}$ plasma power with 10 min of treatment. The reason is that before the maximum value of degree of grafting (24\%), the amounts of peroxides on surface were increased which, in turn, introducing more active sites available for graft polymerization. However, with the continuous increasing in treated power, too many free radicals involved in the self-polymerization reaction that leaded to the homopolymers formation before grafting.

\section{Characteristics Analysis}

ESCA Analysis. ESCA analysis can be used to confirm the reaction of plasma treatment and subsequently graft polymerization. Plasma exposure leads to changes in the elemental composition at the surface of the polymers. The elemental composition at the polymer surface was determined easily with ESCA

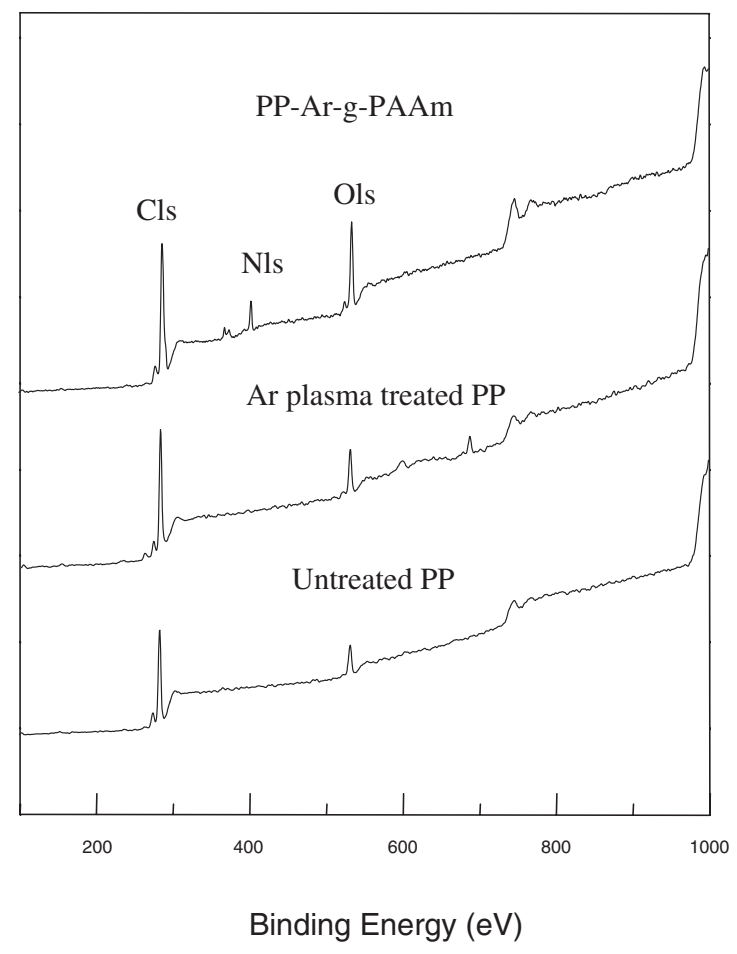

Figure 5. The total energy spectra of untreated PP, Ar plasma treated $(50 \mathrm{~W}, 10 \mathrm{~min}) \mathrm{PP}$, and PP-Ar-g-PAAm fabric (10 wt \% AAm, $30 \mathrm{~min}$ argon preirradiation, UV-induced co-polymerization) from ESCA analysis.

spectra. Figure 5 showed the respective ESCA wide scan for untreated PP, Ar plasma treated $(50 \mathrm{~W}, 10$ min) PP, and PP-Ar-g-PAAm fabric (10 wt \% AAm, 30 min UV-induced co-polymerization, after argon preirradiation). The spectra for the fabric of untreated and argon plasma treated contained a strong peak around at $285.0 \mathrm{eV}$ due to electrons originating from the Cls level and a weak peak at $531.0 \mathrm{eV}$ due to electrons originating from the Ols level and no N1s level spectrum. A significant increase in the relative intensity of the Ols level at $531.0 \mathrm{eV}$ and $399.0 \mathrm{eV}$ of Nls level can be observed from PP-Ar-g-PAAm fabric. The comparison in the Ols level and N1s level spectra between the Ar treated and grafting of PAAm fabric indicates that some nitrogen and oxygen functionalities were generated at the PP fabric surface by the $\mathrm{UV}$-induced co-polymerization of AAm.

Figure 6 showed the Cls level spectra for untreated PP, Ar plasma treated, and AAm grafted on the surface of PP fabric. For the untreated PP fabric, the Cls level spectrum is curve-fitted with two peak components. The main peak at the binding energy of $285.0 \mathrm{eV}$ is due to the $\mathrm{C}-\mathrm{H}$ species, another peak at the binding energy of $286.0 \mathrm{eV}$ is due to the $\mathrm{C}-\mathrm{O}$ species. The surface of PP fabric after Ar plasma treatment contained one additional peak with binding energy at approximately $288.3 \mathrm{eV}$ for the $\mathrm{C}=\mathrm{O}$ species based on the XPS-derived surface stoichiometries. Af- 


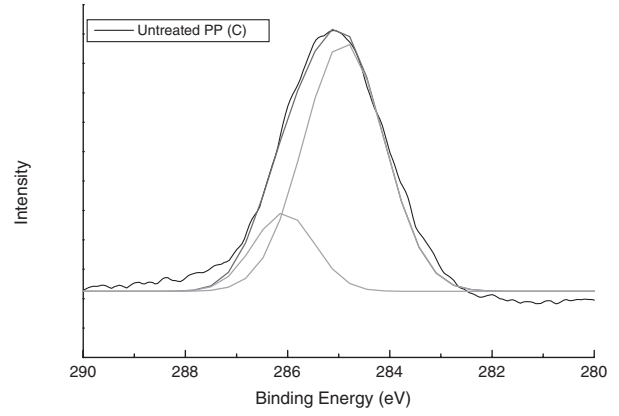

(a)

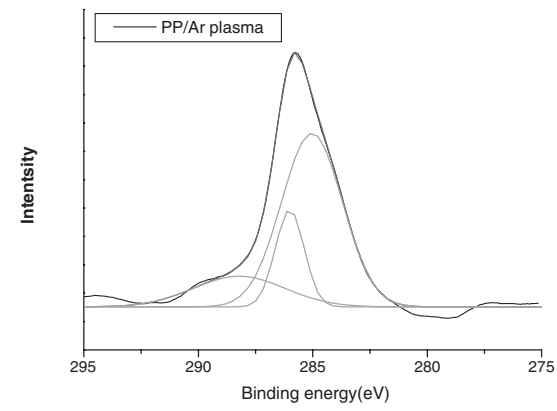

(b)

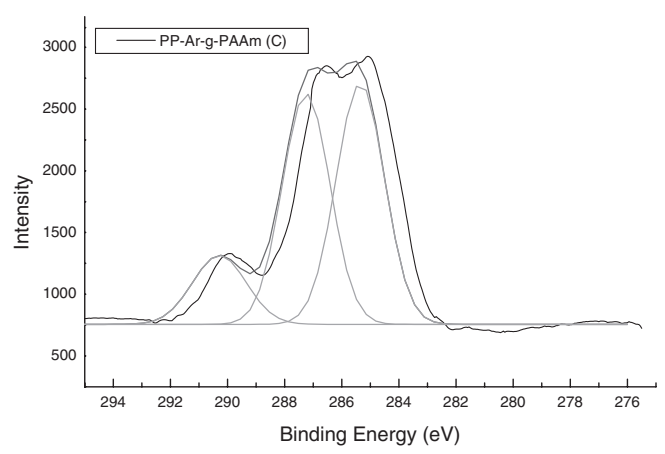

(c)

Figure 6. The C1s spectra of untreated PP and PP-Ar-g-PAAm fabric from ESCA analysis.

Table I. Atomic composition of untreated PP, Ar treated PP, and PP-Ar-g-PAAm fabric determined by ESCA

\begin{tabular}{cccccl}
\hline & \multicolumn{5}{c}{ Chemical Composition (atomic \%) } \\
\cline { 2 - 6 } & $\mathrm{C}$ & $\mathrm{O}$ & $\mathrm{N}$ & $\mathrm{O} / \mathrm{C}$ & $\mathrm{N} / \mathrm{C}$ \\
\hline Origin & 88 & 11 & 1 & 0.13 & 0.011 \\
$*$ & 87 & 12 & 0 & 0.15 & 0 \\
$* *$ & 71 & 18 & 11 & 0.25 & 0.15 \\
\hline
\end{tabular}

*: Ar plasma treated PP: $50 \mathrm{~W}, 10 \mathrm{~min} . * *$ : PP-Ar-g-PAAm: $1000 \mathrm{~W}, 30 \mathrm{~min}, 10 \mathrm{wt} \%$.

ter polymerization with $\mathrm{AAm}$, the peak at $286.7 \mathrm{eV}$ and $289.8 \mathrm{eV}$ were observed on the PP fabric. These peaks are attributed to the $\mathrm{C}-\mathrm{O}$ species at $286.7 \mathrm{eV}$ and $\mathrm{CONH}$ species at $289.8 \mathrm{eV}$, respectively.

Table I listed the atomic composition of PP fabric after various treating conditions by ECSA analysis. From these data, the relative atom composition near the polymer surface could be estimated. For the original and plasma-treated PP fabric, the carbon and oxygen elements were the major composition in analysis. After Ar plasma treated $(10 \mathrm{~min}, 50 \mathrm{~W})$, the atomic composition of $\mathrm{PP}$ fabric in $\mathrm{O} / \mathrm{C}$ increased from 0.13 to 0.15 . A source of the oxygen incorporated into the plasma-treated film is expected that is post-reactions of radicals generated at the film surface with air when taken out from the plasma reactor after plasma treatment. For the PP fabric grafted with $10 \mathrm{wt} \%$ AAm, the composition of nitrogen element was obviously found. That leads to increase in N/C atomic composition from 0 to 0.15 . This proved that AAm was really covalently bound and uniformly distributed onto the PP surface, even after continuous stirring and washing in distilled water.

Scanning Electron Microscopy. When an electron beam impinges the sample surface, low-energy electrons as secondary electron can be detected on within the few $\AA$ of the sample surface by a scintillator detector. Scanning electron morphology has been playing an important role to determine surface morphology of polymers that containing plasma deposited films and photo-induced graft copolymerization. Figures 7 (a) and 7(b) show the surface morphology of the untreated PP fabric and Ar plasma treated PP fabric at $50 \mathrm{~W}$ for $10 \mathrm{~min}$, respectively. The morphology of Ar treated fabric is similar to that of untreated fabric. The surface morphology of PP-Ar-g-PAAm after Ar pretreatment at $50 \mathrm{~W}$ for $10 \mathrm{~min}$ was shown in Figure 7(c). AAm with a concentration of $10 \mathrm{wt} \%$ was grafted on Ar pretreated PP for 30 min. The SEM morphology demonstrates that the AAm was coated on the fabric.

Water Adsorption Ratio of PP Fabric. Although the surface water adsorption ratio can be determined by various methods, the water absorption ratio of PP fabric was employed in this study. Figure 8 shows the absorption ratio of argon-treated PP fabric was striking increased from $3 \%$ to $450 \%$ during $1 \mathrm{~min}$ of plasma treatment then remained a stable value hereafter. This indicates the polar activation surface of 

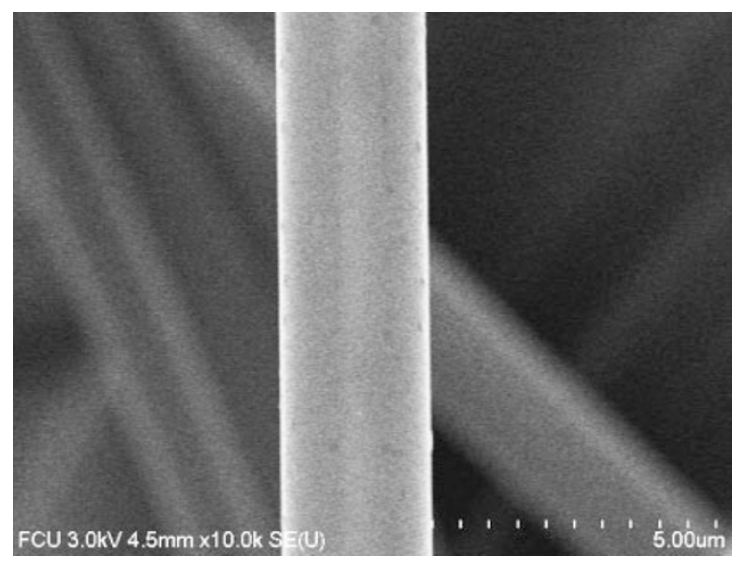

(a)

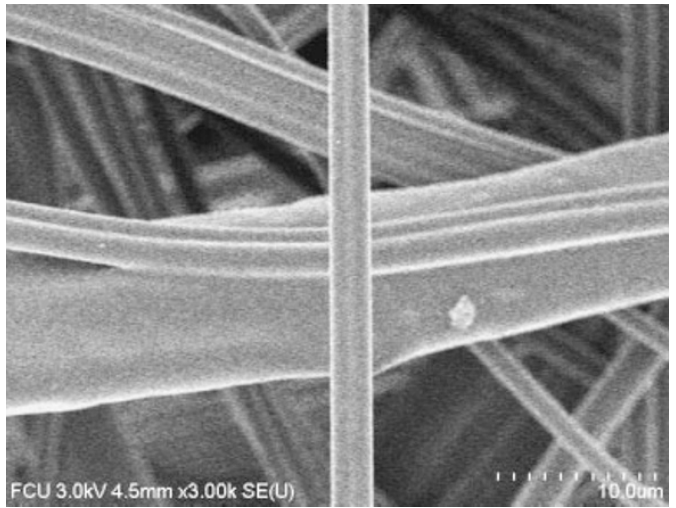

(b)

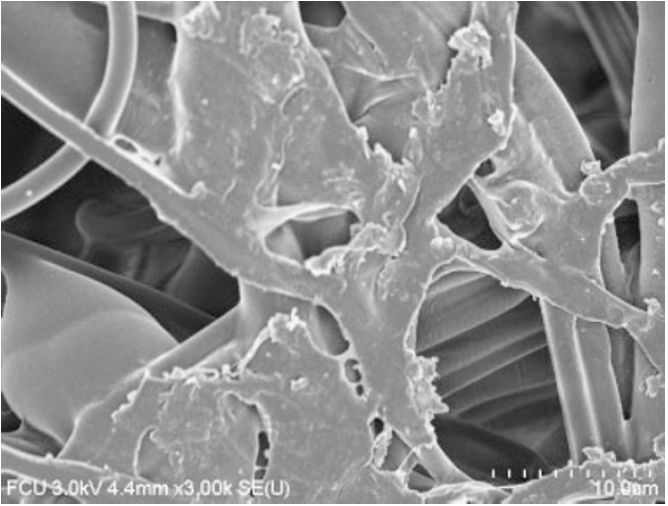

(c)

Figure 7. Morphologies of untreated PP (a), Ar treated PP (b), and PP-Ar-g-PAAm fabric (c) observed from SEM.

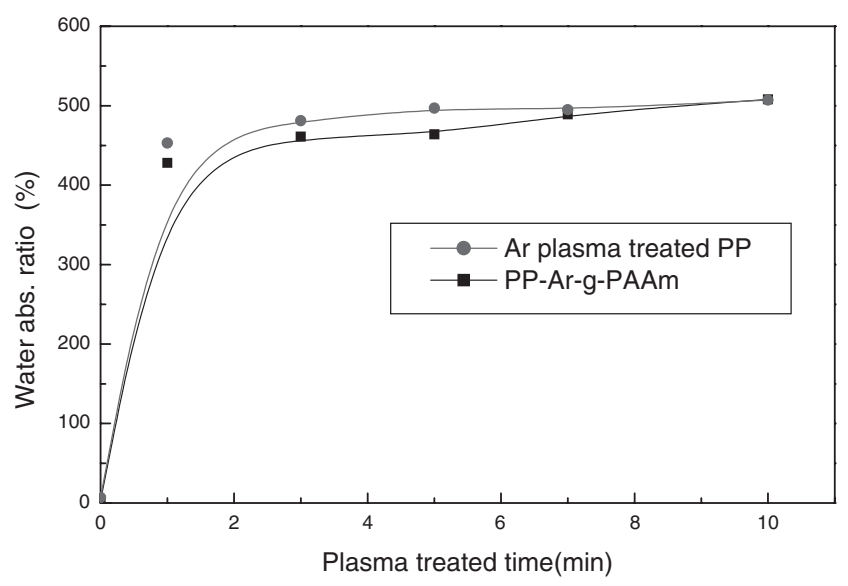

Figure 8. The water adsorption ratio of Ar treated PP and PP-Ar-g-PAAm fabric with the variation of plasma treated time (Plasma treatment: $50 \mathrm{~W}$ ).

PP fabric can be acquired in very short period of time and thus enhancing its surface water adsorption ratio. Similar result was found when PP fabric being grafted with AAm immediately after plasma treatment. For the variation of treated power, the water absorption of argon-treated PP fabric was increased from 3\% to $125 \%$ at treated power up to $15 \mathrm{~W}$, and it needs

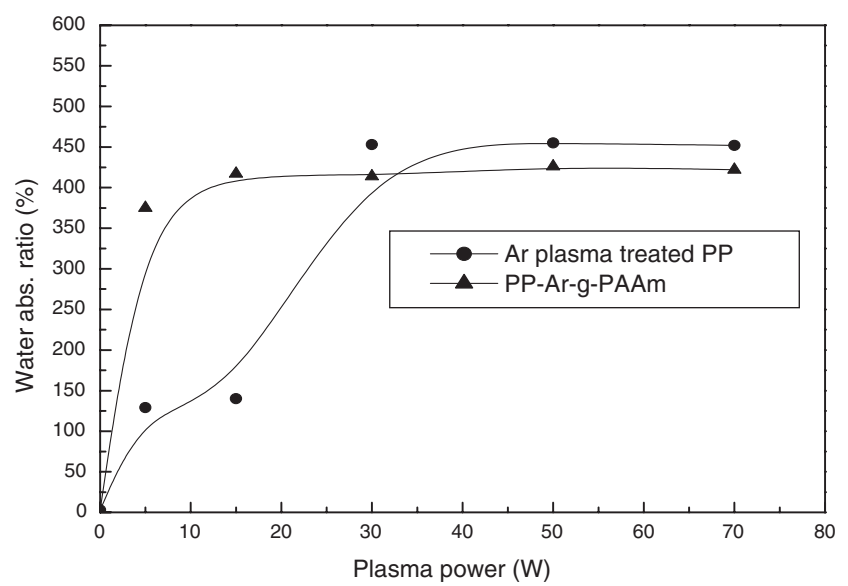

Figure 9. The water adsorption ratio of Ar treated PP and PP-Ar-g-PAAm fabric with the variation of plasma treated power (Plasma treatment: $10 \mathrm{~min}$ ).

$30 \mathrm{~W}$ to reach the saturation (Figure 9). On the other hand, only $5 \mathrm{~W}$ was required for PP-Ar-g-PAAm fabric to reach the saturation of water absorption.

Age Effect on Wettability. Although the plasma activation can make the surface hydrophilic, the stability of the surface against time is deserved for further investigation. As shown in Figure 10, the absorption ra- 


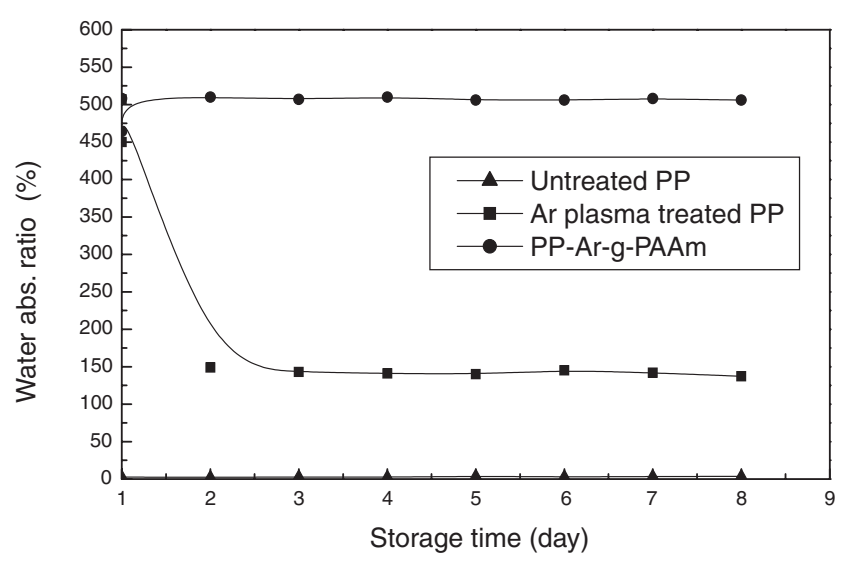

Figure 10. The decay effect of PP fabric after modification with different storage time (Plasma treatment: $50 \mathrm{~W}, 10 \mathrm{~min}$; Grafting: $1000 \mathrm{~W}, 30 \mathrm{~min}$ ).

tio of the sample treated only by argon plasma activation decreased rapidly in the early one or two days of standing time in air. Nevertheless, the absorption ratio of the untreated PP fabric (controlled sample) and after grafted with PAAm remained a stable value of $3 \%$ and $500 \%$, respectively during the storage period. Similar results were found in other systems. ${ }^{18-20}$ The decrease in water absorption ratio of the only plasma-treated PP fabric can be attributed to the polar radicals generated on the surface and subsequently reacted with oxygen atoms in air to form hydrophilic functional groups on surface. However, the partial shift of polar functional groups into the inside of the film due to molecule motions (vibration, winding and spin) would reduce the population of these hydrophilic functional groups on surface. By contrast, the monomer grafted on PP fabric was covalently permanent and can remain nearly the same hydrophilicity even after long time storage for $7 \mathrm{~d}$. The result was similar to N. Inagaki's ${ }^{19}$ report on hydrophilic modification of polyethylene surface by $\mathrm{CO}_{2}, \mathrm{NO}$, and $\mathrm{O}_{2}$ plasma treatments. That pointed out that the hydrophilic property of polyethylene remained after $5 \mathrm{~d}$ of long time storage. Moreover, the fact that the long chain of grating product retarded the mobility of the shift into the inside of the film was another factor contributing its stability.

\section{CONCLUSION}

The surface grafting efficiency of PAAm was obviously affected by the peroxides concentration generated by plasma treatment. From DPPH method, the free radicals could be quantitatively determined. The amounts of peroxides increased with the increasing plasma treated time and treated power. But the results were not monotonous with the increasing plasma treated power on the degree of grafting. An optimum processing condition in this study was determined as $30 \mathrm{~W}$ plasma power with $10 \mathrm{~min}$ of treatment to achieve the maximum surface grafting efficiency.

In addition, the water adsorption ratio of PP fabric can be greatly improved by plasma activation and subsequently grafted polymerization.

Although the surface of PP fabric can be changed from hydrophobic to hydrophilic after plasma treatment, it would obviously age with the storage time. By contrast, the PP surface grafted with AAm can keep their hydrophilic property during the storage time, due to the covalently grafted with hydrophilic monomer.

\section{REFERENCES}

1. L. D. Heather, A. W. StJohn, J. Bi, P. Zientek, R. C. Chatelier, and H. J. Griesser, Surf. Interface Anal., 29, 46 (2000).

2. K. S. Chen, C. W. Chou, S. H. Hsu, and H. R. Lin, Mater. Sci. Forum, 426, 3267 (2003).

3. K. S. Chen, J.-C. Tsai, C.-W. Chou, M. R. Yang, and J. M. Yang, Mater. Sci. Eng., C, 20, 203 (2002).

4. B. O. Aronsson, J. Lausmaa, and B. Kasemo, J. Biomed. Mater. Res., 35, 49 (1997).

5. K. Schröder, A. Meyer-Plath, D. Keller, W. Besch, G. Babucke, and A. Ohl, Contrib. Plasma Phys., 41, 562 (2001).

6. M. R. Yang, K. S. Chen, J. C. Tsai, C. C. Tseng, and S. F. Lin, Mater. Sci. Eng., C, 20, 167 (2002).

7. G. H. Hsiue and W. K. Hang, J. Appl. Polym. Sci., 30, 1023 (1985).

8. Y. Uyama, M. Tadokoro, and Y. Ikada, J. Appl. Polym. Sci., 39, 489 (1990).

9. Y. L. Hsieh and M. Wu, J. Appl. Polym. Sci., 43, 2067 (1991).

10. M. Suzuki, A. Kishida, H. Iwata, and Y. Ikada, Macromolecules, 19, 1804 (1986).

11. I. Kaur, S. Kumar, B. N. Miisra, and G. S. Chauhan, Mater. Sci. Eng., A, 270, 137 (1999).

12. N. Inagaki, S. Tasaka, and Y. Goto, J. Appl. Polym. Sci., 66, 77 (1997).

13. M. Dadsetan, H. Mirzadeh, and N. Sharifi-Sanjani, J. Appl. Polym. Sci., 76, 401 (2000).

14. Z. Ma, C. Gao, J. Yuan, J. Ji, Y. Gong, and J. Shen, J. Appl. Polym. Sci., 85, 2163 (2002).

15. O. H. Kwon, Y. C. Nho, J. H. Jin, M. J. Lee, and Y. M. Lee, J. Appl. Polym. Sci., 72, 659 (1999).

16. J. S. Park, J. H. Kim, Y. C. Nho, and O. H. Kwon, J. Appl. Polym. Sci., 69, 2213 (1998).

17. Y. M. Lee and J. K. Shim, J. Appl. Polym. Sci., 61, 1245 (1996).

18. D. Hegemann, H. Brunner, and C. Oehr, Nucl. Instrum. Methods Phys. Res., Sect. B, 208, 281 (2003).

19. N. Inagaki, S. Tasaka, and K. Hibi, Polym. Prepr., Jpn., 39, 9 (1990).

20. M. L. Steen, A. C. Jordan, and E. R. Fisher, J. Membr. Sci., 204, 341 (2002). 